Acta Crystallographica Section A

Foundations of Crystallography

ISSN 0108-7673

Received 11 March 2009

Accepted 30 April 2009

(C) 2009 International Union of Crystallography Printed in Singapore - all rights reserved

\section{MAD techniques applied to powder data: finding the structure given the substructure}

\author{
Angela Altomare, ${ }^{\mathrm{a}}$ Maria Cristina Burla, ${ }^{\mathrm{d}}$ Corrado Cuocci, ${ }^{\mathrm{a}}$ Carmelo \\ Giacovazzo, ${ }^{a, b}$ Fabia Gozzo, ${ }^{\text {c }}$ Anna Moliterni, ${ }^{a}$ Giampiero Polidori ${ }^{d}$ and Rosanna \\ Rizzi $^{\mathbf{a}}$
}

\begin{abstract}
${ }^{a}$ Istituto di Cristallografia, Sede di Bari, Via G. Amendola 122/o, 70126 Bari, Italy, ${ }^{\mathbf{b}}$ Dipartimento Geomineralogico, Università di Bari, Campus Universitario, Via Orabona 4, 70125 Bari, Italy, ${ }^{\mathbf{c}}$ Swiss Light Source, Paul Scherrer Institute, CH-5232 Villigen PSI, Switzerland, and Dipartimento di Scienze della Terra - Piazza Università, 06100 Perugia, Italy. Correspondence e-mail: carmelo.giacovazzo@ic.cnr.it
\end{abstract}

\begin{abstract}
The joint probability distribution function method is applied to multiplewavelength anomalous dispersion (MAD) powder data. The distributions are calculated by assuming prior knowledge of the scattering intensities at two wavelengths and of the anomalous-scatterer substructure. The method leads to formulas estimating the full structure phases and their reliability. The procedure has been applied to two structures, one unknown and one known; the second was used as a control for the phasing procedure. In spite of the unavoidable peak overlapping in the diffraction pattern, the formulas proved to be very effective. Combined with a new algorithm for phase extension, they enabled the solution of both crystal structures.
\end{abstract}


al., 2002, 2003), cannot be useful for powder data, owing to the fact that Bijvoet pairs cannot be separately estimated;

(b) $\bar{E}_{p}=(1 / 2)\left(E_{p}^{+}+E_{p}^{-*}\right)$ is a more suitable random variable, particularly when $\left|E^{\prime \prime}\right|^{+}$(the anomalous component of $\left|E^{+}\right|$using $f^{\prime \prime}$ as scattering factor) is negligible with respect to $\left|E^{+}\right|$and $\left|E^{-*}\right|$. This occurs when $f^{\prime \prime}$ is sufficiently small and/or when $\left|E_{p}^{+}\right|$and $\left|E_{p}^{-*}\right|$ are sufficiently large. In this case, the phase values $\varphi_{p}^{+}$and $\varphi_{p}^{-*}$ differ by few degrees, and the following approximation holds:

$$
\left|\bar{E}_{p}\right| \simeq(1 / 2)\left(\left|E_{p}^{+}\right|+\left|E_{p}^{-*}\right|\right) .
$$

Accordingly, in paper I the joint probability distribution function $P\left(E_{\mathrm{o} a}, \bar{E}_{1}, \bar{E}_{2}\right)$ was derived, from which the conditional distribution $P\left(R_{\mathrm{o} a} \mid \bar{R}_{1}, \bar{R}_{2}\right)$ and therefore the value of $\left\langle R_{\mathrm{o} a} \mid \bar{R}_{1}, \bar{R}_{2}\right\rangle$ was obtained. This value may be used as input for Patterson and direct methods to find the anomalous-scatterer substructure.

The method was successfully applied to two sets of powder diffraction data, the first originally solved by Janczak \& Kubiak (2002) via a single-crystal experiment [iron(II) phthalocyanine bis(pyridine), $\mathrm{C}_{32} \mathrm{H}_{16} \mathrm{~N}_{8} \mathrm{Fe}\left(\mathrm{C}_{5} \mathrm{H}_{5} \mathrm{~N}\right)_{2}, a=9.576$, $b=19.929, c=9.179 \AA$, $\beta=111.7^{\circ}$, space group $P 2_{1} / c, \mathrm{Z}=2$; from now on indicated as IRON2], the second originally solved by the method described here [trans-dichloridediacetateammine(1-adamantylamine)platinum(IV), $\mathrm{C}_{14} \mathrm{H}_{26}{ }^{-}$ $\mathrm{N}_{2} \mathrm{O}_{4} \mathrm{Cl}_{2} \mathrm{Pt} ; a=11.100, b=13.981, c=6.352 \AA, \alpha=102.3, \beta=$ 104.0, $\gamma=77.5^{\circ}$, space group $P \overline{1}$; from now on indicated as PLAT4].

In this paper we describe a probabilistic method for finding the full structure given the anomalous substructure. The method is derived for the acentric case: the final formulas may be also extended to the centric case, as shown by our applications to IRON2 and PLAT4. To simplify the calculations we neglect the effects of $f^{\prime \prime}$ on $\bar{E}_{p}$.

\section{The joint probability distribution $P\left(R_{1}, R_{2}, R_{3}, \varphi_{1}, \varphi_{2}\right.$,} $\left.\varphi_{3}\right)$

In the following, for simplicity, the variable $\bar{E}_{p}=(1 / 2)\left(E_{p}^{+}+E_{p}^{-*}\right)=\bar{A}_{p}+i \bar{B}_{p}=\bar{R}_{p} \exp \left(i \varphi_{p}\right), \quad p=1,2$, will be denoted by the simpler symbol $E_{p}=A_{p}+i B_{p}$ $=R_{p} \exp \left(i \varphi_{p}\right), p=1,2$. Neglecting the effects of $f^{\prime \prime}$ on $E_{p}$, $p=1,2$, allows us to use the following simplified mathematical model:

$$
\begin{aligned}
& A_{1}=\left[\sum_{j=1}^{N} f_{j}^{0} \cos \left(2 \pi \mathbf{h r}_{j}\right)+\left|\mu_{1}\right| \cos \theta_{1}\right] /\left(\sum_{1}\right)^{1 / 2} \\
& B_{1}=\left[\sum_{j=1}^{N} f_{j}^{0} \sin \left(2 \pi \mathbf{h} \mathbf{r}_{j}\right)+\left|\mu_{1}\right| \sin \theta_{1}\right] /\left(\sum_{1}\right)^{1 / 2} \\
& A_{2}=\left[\sum_{j=1}^{N} g_{j} \cos \left(2 \pi \mathbf{h r}_{j}\right)+\left|\mu_{2}\right| \cos \theta_{2}\right] /\left(\sum_{2}\right)^{1 / 2} \\
& B_{2}=\left[\sum_{j=1}^{N} g_{j} \sin \left(2 \pi \mathbf{h} \mathbf{r}_{j}\right)+\left|\mu_{2}\right| \sin \theta_{2}\right] /\left(\sum_{2}\right)^{1 / 2}
\end{aligned}
$$

$$
\begin{aligned}
& A_{3}=\left[\sum_{j=1}^{a} \Delta f_{j} \cos \left(2 \pi \mathbf{h} \mathbf{r}_{j}\right)+\left|\mu_{3}\right| \cos \theta_{3}\right] /\left(\sum_{3}\right)^{1 / 2} \\
& B_{3}=\left[\sum_{j=1}^{a} \Delta f_{j} \sin \left(2 \pi \mathbf{h} \mathbf{r}_{j}\right)+\left|\mu_{3}\right| \sin \theta_{3}\right] /\left(\sum_{3}\right)^{1 / 2}
\end{aligned}
$$

where

$$
\begin{gathered}
g_{j}=f_{j}^{0}+\Delta f_{j} \text { for } j=1, \ldots a, \quad g_{j}=f_{j}^{0} \text { for } j=a+1, \ldots, N \\
\sum_{1}=\sum_{j=1}^{N} f_{j}^{0^{2}}, \quad \sum_{2}=\sum_{j=1}^{N} g_{j}^{2}=\sum_{1}+\sum_{3}+2 \sum_{13}, \\
\sum_{3}=\sum_{j=1}^{a} \Delta f_{j}^{2}, \quad \sum_{13}=\sum_{j=1}^{a} f_{j}^{0} \Delta f_{j} .
\end{gathered}
$$

$E_{3}=A_{3}+i B_{3}$ represents the normalized structure factor of the model substructure. $\mu_{p}, p=1,2$, is the cumulative complex error arising from measurements and from the full pattern decomposition procedure; $\mu_{3}$ represents the error on the anomalous substructure model. The role of the errors is essential for the usefulness of the mathematical approach. Indeed, if $\mu_{p}, p=1, \ldots, 3$, are assumed to be zeros, the joint probability $P\left(R_{1}, R_{2}, R_{3}, \varphi_{1}, \varphi_{2}, \varphi_{3}\right)$ reduces to a Dirac delta function, owing to the fact that $E_{3}$ exactly equals $E_{2}-E_{1}$.

In accordance with the above definitions, $\Delta f$ may be assumed to be positive or negative: for example, if the first wavelength is chosen far enough from the absorption edge (to make the anomalous corrections to the structure factor negligible) and the second is chosen at the $\operatorname{dip}$ for $f^{\prime}$, then $\Delta f$ is assumed to be negative; vice versa if the first wavelength is at the $\operatorname{dip}$ for $f^{\prime}$ and the second far away from the absorption edge then $\Delta f$ is assumed to be positive.

In polar variables, the joint probability distribution $P\left(R_{1}, R_{2}, R_{3}, \varphi_{1}, \varphi_{2}, \varphi_{3}\right)$ is given by

$$
\begin{aligned}
P\left(R_{1},\right. & \left.R_{2}, R_{3}, \varphi_{1}, \varphi_{2}, \varphi_{3}\right) \\
= & (\operatorname{det} \mathbf{K})^{-1 / 2} \prod_{j=1}^{3}\left(\pi^{-1} R_{j} / e_{j}\right) \\
& \quad \times \exp \left[-\sum_{i=1}^{3} \Lambda_{i i} R_{i}^{2}-2 \sum_{i, j=1}^{3} \Lambda_{i j} R_{i} R_{j} \cos \left(\varphi_{i}-\varphi_{j}\right)\right],
\end{aligned}
$$

where

$$
\begin{gathered}
e_{j}=\left(1+\sigma_{j}^{2}\right), j=1, \ldots, 3, \quad \sigma_{j}^{2}=\left\langle\left|\mu_{j}\right|^{2}\right\rangle / \sum_{j}, j=1, \ldots, 3, \\
\sum_{12}=\sum_{1}+\sum_{13}, \quad \sigma_{12}=\sum_{12} /\left(\sum_{1} \sum_{2}\right)^{1 / 2} \\
\sigma_{13}=\sum_{13} /\left(\sum_{1} \sum_{3}\right)^{1 / 2}, \\
\sum_{23}=\sum_{j=1}^{a} g_{j} \Delta f_{j}=\sum_{13}+\sum_{3}, \quad \sigma_{23}=\sum_{23} /\left(\sum_{2} \sum_{3}\right)^{1 / 2}, \\
\mathbf{K}=\left|\begin{array}{cc}
\mathbf{L} & 0 \\
0 & \mathbf{L}
\end{array}\right|, \quad \mathbf{K}^{-1}=\left|\begin{array}{cc}
\mathbf{L}^{-1} & 0 \\
0 & \mathbf{L}^{-1}
\end{array}\right|, \\
\operatorname{det} \mathbf{K}=\left(e_{1} e_{2} e_{3}-e_{1} \sigma_{23}^{2}-e_{2} \sigma_{13}^{2}-e_{3} \sigma_{12}^{2}+2 \sigma_{12} \sigma_{13} \sigma_{23}\right)^{2} /\left(e_{1} e_{2} e_{3}\right)^{2}
\end{gathered}
$$




$$
\begin{aligned}
& \mathbf{L}=\left|\begin{array}{ccc}
1 & \sigma_{12} /\left(e_{1} e_{2}\right)^{1 / 2} & \sigma_{13} /\left(e_{1} e_{3}\right)^{1 / 2} \\
\sigma_{12} /\left(e_{1} e_{2}\right)^{1 / 2} & 1 & \sigma_{23} /\left(e_{2} e_{3}\right)^{1 / 2} \\
\sigma_{13} /\left(e_{1} e_{3}\right)^{1 / 2} & \sigma_{23} /\left(e_{2} e_{3}\right)^{1 / 2} & 1
\end{array}\right|, \\
& \Lambda_{11}=\frac{1}{e_{1} e_{2} e_{3} \operatorname{det} \mathbf{L}}\left(1-\frac{\sum_{23}^{2}}{\sum_{2} \sum_{3}}\right), \\
& \Lambda_{22}=\frac{1}{e_{1} e_{2} e_{3} \operatorname{det} \mathbf{L}}\left(1-\frac{\sum_{13}^{2}}{\sum_{1} \sum_{3}}\right) \text {, } \\
& \Lambda_{33}=\frac{1}{e_{1} e_{2} e_{3} \operatorname{det} \mathbf{L}}\left(1-\frac{\sum_{12}^{2}}{\sum_{1} \sum_{2}}\right) \text {, } \\
& \Lambda_{12}=\frac{1}{e_{1} e_{2} e_{3} \operatorname{det} \mathbf{L}}\left[\frac{\sum_{13}^{2}+\sum_{3} \sum_{13}\left(1-e_{3}\right)-\sum_{1} \sum_{3} e_{3}}{\left(\sum_{1} \sum_{2}\right)^{1 / 2} \sum_{3}}\right] \text {, } \\
& \Lambda_{13}=\left[1 /\left(e_{1} e_{2} e_{3} \operatorname{det} \mathbf{L}\right)\right]\left[\sum_{13}^{2}\left(1-2 e_{2}\right)+\sum_{3} \sum_{13}\left(1-e_{2}\right)\right. \\
& \left.+\sum_{1} \sum_{13}\left(1-e_{2}\right)+\sum_{1} \sum_{3}\right] /\left[\left(\sum_{1} \sum_{3}\right)^{1 / 2} \sum_{2}\right] \\
& =\left[1 /\left(e_{1} e_{2} e_{3} \operatorname{det} \mathbf{L}\right)\right]\left[\sum_{1} \sum_{3}-e_{2} \sum_{13}^{2}+\sum_{13}\left(1-e_{2}\right)\right. \\
& \left.\times\left(\sum_{13}+\sum_{1}+\sum_{3}\right)\right] /\left[\left(\sum_{1} \sum_{3}\right)^{1 / 2} \sum_{2}\right] \\
& =\left[1 /\left(e_{1} e_{2} e_{3} \operatorname{det} \mathbf{L}\right)\right]\left[\left(\sum_{1} \sum_{3}-\sum_{13}^{2}\right)\right. \\
& \left.+\left(1-e_{2}\right) \sum_{2} \sum_{13}\right] /\left[\left(\sum_{1} \sum_{3}\right)^{1 / 2} \sum_{2}\right] \text {, } \\
& \Lambda_{23}=\frac{1}{e_{1} e_{2} e_{3} \operatorname{det} \mathbf{L}}\left[\frac{\sum_{13}^{2}-\sum_{1} \sum_{3} e_{1}+\sum_{1} \sum_{13}\left(1-e_{1}\right)}{\left(\sum_{2} \sum_{3}\right)^{1 / 2} \sum_{1}}\right] .
\end{aligned}
$$

\section{Phasing the crystal-structure reflections}

In order to associate phase values to the full structure reflections we need to derive the conditional distributions $P\left(\varphi_{1} \mid R_{1}, R_{2}, R_{3}, \varphi_{3}\right)$ or $P\left(\varphi_{2} \mid R_{1}, R_{2}, R_{3}, \varphi_{3}\right)$. Such distributions cannot be obtained from equation (1) without some approximations. In accordance with Giacovazzo \& Siliqi (2001) the approximation $\varphi_{1} \simeq \varphi_{2}$ may be introduced, which is fully justified if $R_{1}$ and $R_{2}$ are sufficiently large. Then equation (1) becomes

$$
\begin{aligned}
P\left(R_{1}, R_{2}, R_{3}, \varphi_{1}, \varphi_{3}\right) \simeq & 2 \pi \prod_{j=1}^{3}\left(\pi^{-1} R_{j} / e_{j}\right)(\operatorname{det} \mathbf{K})^{-1 / 2} \\
& \times \exp \left[-\sum_{i=1}^{3} \Lambda_{i i} R_{i}^{2}+G \cos \left(\varphi_{1}-\varphi_{3}\right)\right],
\end{aligned}
$$

where

$$
G=-2\left(\Lambda_{13} R_{1}+\Lambda_{23} R_{2}\right) R_{3},
$$

and the following von Mizes conditional distribution arises:

$$
P\left(\varphi_{1} \mid R_{1}, R_{2}, R_{3}, \varphi_{3}\right) \simeq\left[2 \pi I_{0}(G)\right]^{-1} \exp \left[G \cos \left(\varphi_{1}-\varphi_{3}\right)\right] .
$$

From equation (2) the following rule may be deduced: if $\Lambda_{13} R_{1}+\Lambda_{23} R_{2}$ is negative the probabilistic relation $\varphi_{1} \simeq \varphi_{3}$ is supported. If $\Lambda_{13} R_{1}+\Lambda_{23} R_{2}$ is positive the relation $\varphi_{1} \simeq \varphi_{3}+\pi$ is more probable. The probabilities are sharper when $R_{3}$ is large.

To provide a simple qualitative insight into the signs and the moduli of the $\Lambda_{i j}$ we simplify their numerators by assuming that $e_{j}$ for $j=1,2,3$ are sufficiently close to unity: we find

$$
\begin{aligned}
& \Lambda_{12}=-\frac{1}{e_{1} e_{2} e_{3} \operatorname{det} \mathbf{L}} \frac{Z_{\mathrm{c}}}{\left(\sum_{1} \sum_{2}\right)^{1 / 2} \sum_{3}}, \\
& \Lambda_{13}=\frac{1}{e_{1} e_{2} e_{3} \operatorname{det} \mathbf{L}} \frac{Z_{\mathrm{c}}}{\left(\sum_{1} \sum_{3}\right)^{1 / 2} \sum_{2}}, \\
& \Lambda_{23}=-\frac{1}{e_{1} e_{2} e_{3} \operatorname{det} \mathbf{L}} \frac{Z_{\mathrm{c}}}{\left(\sum_{2} \sum_{3}\right)^{1 / 2} \sum_{1}},
\end{aligned}
$$

where

$$
Z_{\mathrm{c}}=\sum_{1} \sum_{3}-\sum_{13}^{2}
$$

If, as frequently occurs, the scattering power of the anomalous substructure is sufficiently smaller than that of the full structure, then:

(i) $Z_{\mathrm{c}}>0$;

(ii) $\Lambda_{13}$ is positive while $\Lambda_{12}$ and $\Lambda_{23}$ are negative;

(iii) $\Lambda_{12} / \Lambda_{13}=-\left(\sum_{2} / \sum_{3}\right)^{1 / 2}, \Lambda_{13} / \Lambda_{23}=-\left(\sum_{1} / \sum_{2}\right)^{1 / 2}$, $\Lambda_{12} / \Lambda_{23}=\left(\sum_{1} / \sum_{3}\right)^{1 / 2}$

(iv) $\Lambda_{23} \simeq-\Lambda_{13}$ and equation (2) reduces to

$$
G \simeq 2 \Lambda_{13}\left(R_{2}-R_{1}\right) R_{3} .
$$

In other words, under the above assumptions, the reliability of the phase indication $\varphi_{1} \simeq \varphi_{3}$ depends on the modulus $R_{3}$ and on the difference $R_{2}-R_{1}$ [see Giacovazzo \& Siliqi (2001) for a related formula]: $\varphi_{1} \simeq \varphi_{3}$ if $R_{2}>R_{1}, \varphi_{1} \simeq \varphi_{3}+\pi$ if $R_{2}<R_{1}$.

It is worth noting that if $\Delta f$ is assumed to be negative (i.e., when the second wavelength is chosen at the $\operatorname{dip}$ for $f^{\prime}$ ), then

$$
\sum_{1}>\sum_{2} \text { and }\left|\Lambda_{12}\right|>\left|\Lambda_{13}\right|>\left|\Lambda_{23}\right| .
$$

If $\Delta f$ is assumed to be positive (i.e., when the first wavelength is at the $\operatorname{dip}$ for $f^{\prime}$ ), then

$$
\sum_{2}>\sum_{1} \text { and }\left|\Lambda_{12}\right|>\left|\Lambda_{23}\right|>\left|\Lambda_{13}\right| .
$$

The above results are obtained by supposing $\varphi_{1} \simeq \varphi_{2}$. This is not always true, and therefore we look for a more accurate approach. We first integrate equation (1) on $\varphi_{2}$ : we have

$$
\begin{aligned}
P\left(R_{1},\right. & \left.R_{2}, R_{3}, \varphi_{1}, \varphi_{3}\right) \\
= & 2 \pi \prod_{j=1}^{3}\left(\pi^{-1} R_{j} / e_{j}\right)(\operatorname{det} \mathbf{K})^{-1 / 2} R_{1} R_{2} R_{3} \\
& \times \exp \left[-\sum_{i=1}^{3} \Lambda_{i i} R_{i}^{2}-2 \Lambda_{13} R_{1} R_{3} \cos \left(\varphi_{1}-\varphi_{3}\right)\right] I_{0}\left(G_{13}\right),
\end{aligned}
$$

where

$$
G_{13}=2 R_{2}\left[\Lambda_{12}^{2} R_{1}^{2}+\Lambda_{23}^{2} R_{3}^{2}+2 \Lambda_{12} \Lambda_{23} R_{1} R_{3} \cos \left(\varphi_{1}-\varphi_{3}\right)\right]^{1 / 2} .
$$

We then use the following approximation (Giacovazzo, 1979):

$$
\begin{aligned}
& I_{0}\left[Q_{1}^{2}+Q_{2}^{2}+2 Q_{1} Q_{2} \cos (\phi-\vartheta)\right]^{1 / 2} \\
& \quad=\left[I_{0}\left(Q_{1}\right) I_{0}\left(Q_{2}\right) / I_{0}(Q)\right] \exp [Q \cos (\phi-\vartheta)],
\end{aligned}
$$

where 


$$
D_{1}(Q)=D_{1}\left(Q_{1}\right) D_{1}\left(Q_{2}\right)
$$

Accordingly

$$
\begin{aligned}
P\left(R_{1}, R_{2}, R_{3}, \varphi_{1}, \varphi_{3}\right) & \\
= & 2 \pi \prod_{j=1}^{3}\left(\frac{\pi^{-1} R_{j}}{e_{j}}\right) \frac{I_{0}\left(2 \Lambda_{12} R_{1} R_{2}\right) I_{0}\left(2 \Lambda_{23} R_{2} R_{3}\right)}{I_{0}\left(G_{13}\right)} \\
& \times(\operatorname{det} \mathbf{K})^{-1 / 2} \exp \left[-\sum_{i=1}^{3} \Lambda_{i i} R_{i}^{2}+S \cos \left(\varphi_{1}-\varphi_{3}\right)\right],
\end{aligned}
$$

where

$$
S=\left(Q-2 \Lambda_{13} R_{1} R_{3}\right)
$$

and

$$
D_{1}(Q)=D_{1}\left(2 \Lambda_{12} R_{1} R_{2}\right) D_{1}\left(2 \Lambda_{23} R_{2} R_{3}\right) .
$$

Since $\Lambda_{12}$ and $\Lambda_{23}$ have the same sign, $Q$ is always positive. Let us now calculate

$$
P\left(\varphi_{1} \mid R_{1}, R_{2}, R_{3}, \varphi_{3}\right)=\left[2 \pi I_{0}(S)\right]^{-1} \exp \left[S \cos \left(\varphi_{1}-\varphi_{3}\right)\right]
$$

from which

$$
\varphi_{1} \simeq \varphi_{3} \text { if } S>0, \quad \varphi_{1} \simeq \varphi_{3}+\pi \text { if } S<0 .
$$

To understand equations (5)-(8) let us suppose that $2 \Lambda_{12} R_{1} R_{2}$ is very large and negative. Then, according to equation (6), $D_{1}\left(2 \Lambda_{12} R_{1} R_{2}\right) \simeq-1, Q \simeq-2 \Lambda_{23} R_{2} R_{3}$, and $S$ reduces to $G$, as defined by equation (2). The above result makes it clear that the assumption $\varphi_{1} \simeq \varphi_{2}$ is only justified if $2 \Lambda_{12} R_{1} R_{2}$ is large enough.

If $R_{1}$ and $R_{3}$ are large while $R_{2}$ is small, then $Q<2 \Lambda_{13} R_{1} R_{3}$ and the relation $\varphi_{1} \simeq \varphi_{3}+\pi$ is suggested, in accordance with equation (4).

Let us now suppose that $2 \Lambda_{23} R_{2} R_{3}$ is very large and negative, as occurs in the most favourable cases. Then $D_{1}\left(2 \Lambda_{23} R_{2} R_{3}\right) \simeq-1$ and $Q \simeq-2 \Lambda_{12} R_{1} R_{2}:$ in this case we have

$$
S=-2 \Lambda_{12} R_{1} R_{2}-2 \Lambda_{13} R_{1} R_{3}=-2\left(\Lambda_{13} R_{3}+\Lambda_{12} R_{2}\right) R_{1} .
$$

The most probable phase relationship will be $\varphi_{1} \simeq \varphi_{3}+\pi$ or $\varphi_{1} \simeq \varphi_{3}$ according to whether $\Lambda_{13} R_{3}+\Lambda_{12} R_{2}>0$ or $\Lambda_{13} R_{3}+\Lambda_{12} R_{2}<0$, respectively. As we see, in disagreement with equation (2), the choice between the two alternatives no longer depends on the difference between $R_{1}$ and $R_{2}$ (via the value of $\Lambda_{13} R_{1}+\Lambda_{23} R_{2}$ ), but on the difference between $R_{2}$ and $R_{3}$ (via the value of $\Lambda_{13} R_{3}+\Lambda_{12} R_{2}$ ).

Since equations (5)-(8) have general validity, it is expected that they can provide better estimates than equations (2) and (3).

\section{Simplified expressions for phase estimations}

The phasing formulas described in the preceding section critically depend on the errors $e_{1}, e_{2}, e_{3}$. For example, $\Lambda_{12}, \Lambda_{13}$ and $\Lambda_{23}$ values vary with $e_{3}, e_{2}, e_{1}$ : as a consequence $G$ and $S$ values, as defined by equations (2) and (5), respectively, will depend not only on $R_{1}, R_{2}, R_{3}$, but also on $e_{1}, e_{2}, e_{3}$. In particular, $G$ and $S$ may change their sign in specific circumstances. Such behaviour is in principle correct: however peak overlapping, possible preferred orientation and difficult background definition can make the error estimation quite a serious problem. In particular the Le Bail (Le Bail et al., 1988) decomposition approach does not associate weights to decomposed intensities; the Pawley method, on the other hand, provides weights which may be used in the structurerefinement step (David, 2004). Suitable weights for leastsquares refinement were also described by Altomare et al. (2006).

In general, full control of the errors in powder crystallography is more difficult than for single-crystal data. Accordingly, it may be useful to simplify the phase relationships derived in $\$ 4$ in order to make them less critically dependent on the errors and, simultaneously, simpler and faster. In Appendix $A$ we show that

$$
e_{1} e_{2} e_{3} \operatorname{det}(\mathbf{L}) \simeq e_{3}\left(e_{1} e_{2}-1\right) Z_{\mathrm{c}} \sum_{2} /\left(\sum_{1} \sum_{2} \sum_{3}\right) .
$$

Introducing the above relation in equation (2) gives

$$
\begin{aligned}
G & =-2\left(\Lambda_{13} R_{1}+\Lambda_{23} R_{2}\right) R_{3} \\
& =\frac{2 \sum_{3}^{1 / 2}}{e_{3}\left(e_{1} e_{2}-1\right) \sum_{2}}\left(\sum_{2}^{1 / 2} R_{2}-\sum_{1}^{1 / 2} R_{1}\right) R_{3} \\
& =\frac{2}{e_{3}\left(e_{1} e_{2}-1\right) \sum_{2}}\left(\left|F_{2}\right|-\left|F_{1}\right|\right)\left|F_{3}\right| \\
& =\frac{2}{e_{3}\left(e_{1} e_{2}-1\right)}\left(R_{2}-R_{1}^{\prime}\right) R_{3}^{\prime},
\end{aligned}
$$

where $R_{2}^{\prime}$ and $R_{3}^{\prime}$ are structure factors pseudonormalized with respect to $\sum_{2}^{1 / 2}$. Equation (9) shows that the reliability parameter $G$ assumes a very simple algebraic form when expressed in terms of structure factors or in terms of pseudonormalized structure factors. The expected value of $\varphi_{1}$ depends on the difference $\left(\left|F_{2}\right|-\left|F_{1}\right|\right)$; its reliability increases with $\left|F_{3}\right|$ and is modulated by the error function $\left[e_{3}\left(e_{1} e_{2}-1\right) \sum_{2}\right]^{-1}$. The larger $e_{1}, e_{2}, e_{3}$ are, the smaller the reliability of the phase indication is. Equation (9) also suggests that the accurate scaling of the intensities collected at the two wavelengths is a necessary condition for the success of the approach.

If we apply the same technique to simplify equations (5)-(7) we find that $S$ is still defined by them, but now

$$
\begin{aligned}
& 2 \Lambda_{12} R_{1} R_{2}=-\frac{2}{e_{3}\left(e_{1} e_{2}-1\right) \sum_{2}}\left|F_{1} F_{2}\right|=-\frac{2}{e_{3}\left(e_{1} e_{2}-1\right)} R_{1}^{\prime} R_{2}, \\
& 2 \Lambda_{13} R_{1} R_{3}=\frac{2}{e_{3}\left(e_{1} e_{2}-1\right) \sum_{2}}\left|F_{1} F_{3}\right|=\frac{2}{e_{3}\left(e_{1} e_{2}-1\right)} R_{1}^{\prime} R_{3}^{\prime},
\end{aligned}
$$

$2 \Lambda_{23} R_{2} R_{3}=-\frac{2}{e_{3}\left(e_{1} e_{2}-1\right) \sum_{2}}\left|F_{2} F_{3}\right|=-\frac{2}{e_{3}\left(e_{1} e_{2}-1\right)} R_{2} R_{3}^{\prime}$.

\section{Applications}

The probabilistic formulas derived in $\S \S 4$ and 5 were implemented in a modified version of EXPO2004 (Altomare et al., 
2004): the procedure was applied to IRON2 and PLAT4 using as prior information the anomalous substructures obtained via the approach described in paper I. In particular, we used in the formulas the experimental $\left|F_{1}\right|$ and $\left|F_{2}\right|$ values obtained by the EXPO2004 default full pattern decomposition procedure (Le Bail approach), and the $\left|F_{3}\right|$ values calculated from the known substructures. To be consistent, we report in Table 1 the experimental data quoted in Table 1 of paper I, along with the experimental data resolution (RES) for each wavelength.

In order to estimate the phases $\varphi_{1}$ we used both equations (2)-(3) and (5)-(7) derived in $\$ 4$ and the corresponding simplified expressions [via equations (9) and (10)] obtained in $\S 5$. Since the two approaches did not show remarkable differences, for the sake of simplicity we will describe the results obtained via the simplified formulas.

The full phasing process will be described as steps.

(1) Phasing via anomalous dispersive data. In paper I the dispersive data were used to locate the anomalous scatterers: for IRON2 the Fe atom was found in the special position $(0,0$, 0 ) and for PLAT4 Pt was located at $(0.667,0.311,0.049)$.

We applied equation (9) to the 332 reflections lying in both measured powder patterns for IRON2 (i.e. up to $1.72 \AA$ resolution): in the absence of a sound weighting criterion we assumed $e_{1}, e_{2}, e_{3}$ constantly equal to 1.05 .168 reflections were phased, with average phase error (with respect to the phases calculated from the refined crystal structure) $\langle|\Delta \varphi|\rangle=25^{\circ}$ [it may be worth noting that the 168 phased reflections satisfy the relation $k+l=2 n$ : indeed, since $\mathrm{Fe}$ is on an inversion centre, it does not contribute to the reflections with $k+l=2 n+1$, no matter what wavelength is used]. The cumulative distribution versus $G$ of the phase error is shown in Table 2 .

The calculations made for IRON2 were repeated for PLAT4. We applied equation (9) to the 1289 reflections lying in both measured powder patterns (i.e. up to $1.14 \AA$ resolution): 1228 reflections were phased with $\langle|\Delta \varphi|\rangle=21^{\circ}$. In Table 3 we show the cumulative distribution versus $G$ of the phase error.

Tables 2 and 3 indicate that the phase error distribution has the fortunate property that the wrong phase estimates are mostly confined to low values of $G$.

The supplementary calculations necessary to derive equations (5)-(7) are aimed at eliminating the assumption $\varphi_{1} \simeq \varphi_{2}$, which was at the basis of equations (2) and (3). Equations (5)(7) were then simplified via the relationships in equation (10) to take into account the uncertainty of the weighting criterion. We applied the simplified version of equations (5)-(7) to IRON2 and PLAT4. The cumulative phase error distributions versus $S$ are shown in columns 6 and 7 of Table 2 and in columns 6 to 9 of Table 3: the use of the reliability parameter $S$ provides slightly better results (i.e. the total number of wrong phase estimates is slightly smaller).

(2) Phase extension. Dispersive scattering allowed us to phase IRON2 reflections up to $1.72 \AA$ and PLAT4 reflections up to $1.14 \AA$. To refine and extend phases we first applied the standard EXPO Fourier recycling procedure (Altomare et al., 2006). For PLAT4 the phasing process was extended up to
Table 1

Experimental data.

For each test structure the following information is given: selected wavelengths, literature values for $\Delta f$ and $f^{\prime \prime}$ expected at the remote and at the $\operatorname{dip}$ (for $f^{\prime}$ ) wavelengths, the experimental $2 \theta$ ranges (RAN), the corresponding number of reflections (NREF), and the experimental data resolution (RES).

\begin{tabular}{llrllrl}
\hline Code & $\lambda(\AA)$ & \multicolumn{1}{c}{$\Delta f$} & $f^{\prime \prime}$ & RAN $(2 \theta)\left({ }^{\circ}\right)$ & NREF & RES $(\AA)$ \\
\hline IRON2 & 1.07 & 0.0 & 0.0 & $1.0-40.9$ & 464 & 1.54 \\
IRON2 & 1.74 & -8.0 & 0.6 & $1.0-60.9$ & 332 & 1.72 \\
PLAT4 & 0.95 & 0.0 & 0.0 & $1.0-55.9$ & 1826 & 1.02 \\
PLAT4 & 1.07 & -16.0 & 4.0 & $1.0-55.9$ & 1289 & 1.14 \\
\hline
\end{tabular}

$1.02 \AA$ and the procedure ended with $\langle|\Delta \varphi|\rangle=22^{\circ}$ for 1826 reflections: the corresponding electron density showed 15 out of 23 peaks with $\langle d\rangle=0.27 \AA(\langle d\rangle$ is the average distance between the experimental and the published atomic positions).

The application of the same techniques to IRON2 (phase extension from 1.72 to $1.54 \AA$ ) provided a mean phase error $\langle|\Delta \varphi|\rangle=84^{\circ}$ for 464 reflections. No useful electron-density map was provided: in practice the standard EXPO Fourier recycling procedure spoils the extremely good phase estimates obtained via anomalous dispersive pairs. It was, however, noticed that the reflections with $k+l=2 n+1$ (to which Fe does not contribute) were all phased with a very small reliability parameter. We then introduced the following new algorithm:

(a) Triplet invariants are calculated among the strongest reflections $(\mathrm{NSTRONG}=220$ ).

(b) A tangent procedure relying on the triplets in $(a)$ is applied, by including into the starting set the phases of the reflections with $k+l=2 n$ and five symbols, represented by a magic integer sequence (Main, 1978). The procedure automatically selects the symbols among the subset of reflections for which $k+l=2 n+1$. The corresponding trial phases are used for extending the phasing process: spoiling of the original phase estimates is avoided by holding fixed the 100 more reliable phases determined via anomalous dispersion effects.

(c) The phases obtained at the end of step (b) are merged with the estimates obtained by equations (5)-(7).

(d) The resolution bias correction procedure (Altomare et al., 2008) is applied to the phases obtained at step $(c)$. The algorithm cyclically corrects the truncation effects in any calculated electron-density map.

At the end of step $(d) 464$ phases were determined with $\langle|\Delta \varphi|\rangle=34^{\circ}$. In the electron-density map 27 peaks out of 27 could be found with an average distance from the correct positions $\langle d\rangle=0.31 \AA$. It is worth noting the important role of the resolution bias correction procedure: if step $(d)$ is omitted, then 261 phases were determined with $\langle|\Delta \varphi|\rangle=22^{\circ}$ and only 15 peaks in the final electron-density map are correctly positioned, with $\langle d\rangle=0.34 \AA$.

(3) Structure completion and refinement. The Rietveld method (Rietveld, 1969) was used to complete and refine both test structures. 
Table 2 'Negative relationships' and 'Positive relationships' refer to reflections for which $G$ [and therefore $\left.\left\langle\cos \left(\varphi_{1}-\varphi_{3}\right)\right\rangle\right]$ is negative or positive, respectively. For each value of ARG the number of relationships with $|G|>\mathrm{ARG}$ (No.) and the corresponding average phase error $\langle|\Delta \varphi|\rangle$ are given. In columns 6 and 7 equations (5) to (7), simplified via equations (10), are applied. In this case only negative (i.e. reflections for which $S$ is negative) relationships are obtained. For each value of ARG the number of relationships with $|S|>$ ARG (No.) and the corresponding average phase error $\langle|\Delta \varphi|\rangle$ are given.

\begin{tabular}{|c|c|c|c|c|c|c|}
\hline \multirow[b]{3}{*}{ ARG } & \multicolumn{4}{|c|}{ Equations (2) and (3) } & \multirow{2}{*}{\multicolumn{2}{|c|}{$\begin{array}{l}\text { Equations (5) to (7) } \\
\text { Negative relationships }\end{array}$}} \\
\hline & \multicolumn{2}{|c|}{ Negative relationships } & \multicolumn{2}{|c|}{ Positive relationships } & & \\
\hline & No. & $\langle|\Delta \varphi|\rangle\left(^{\circ}\right)$ & No. & $\langle|\Delta \varphi|\rangle\left(^{\circ}\right)$ & No. & $\langle|\Delta \varphi|\rangle\left(^{\circ}\right)$ \\
\hline 0.0 & 143 & 5 & 25 & 137 & 168 & 11 \\
\hline 2.0 & 121 & 4 & 12 & 150 & 156 & 9 \\
\hline 4.0 & 95 & 4 & 8 & 180 & 141 & 6 \\
\hline 6.0 & 67 & 3 & 2 & 180 & 117 & 6 \\
\hline 8.0 & 50 & 4 & & & 95 & 4 \\
\hline 10.0 & 35 & 0 & & & 81 & 2 \\
\hline 12.0 & 24 & 0 & & & 59 & 3 \\
\hline 14.0 & 14 & 0 & & & 41 & 4 \\
\hline
\end{tabular}

Cumulative distributions of the phase error versus ARG for IRON2.

In columns 2 to 5 equations (2) and (3), simplified via equation (9), are applied. The columns

$$
\begin{aligned}
\mathrm{RC} & =\left(\sum_{h}|| y_{h}|-| x_{h}||\right) / \sum_{h}\left|y_{h}\right| \\
\text { and } \mathrm{RCS} & =\left(\sum_{h}\left|y_{h}\right|-\left|x_{h}\right|\right) / \sum_{h}\left|y_{h}\right| .
\end{aligned}
$$

The first is the classical crystallographic residual, the second takes into account the signs of the differences between $x$ and $y$. In Fig. 1 we show the $\mathrm{RC}_{i}$ and $\mathrm{RCS}_{i}$ values for the pairs $\left(R_{i \mathrm{t}}, R_{i \mathrm{~d}}\right)$ for $i=1,2$, versus the interplanar spacing $d$ : they are calculated for batches of 100 reflections sharing the same $2 \theta$ interval. $R_{i \mathrm{t}}$ is the normalized structure-factor modulus as calculated from the refined structure, $R_{i \mathrm{~d}}$ is that obtained via full pattern decomposition. For both wavelengths $\mathrm{RC}_{i}$ increases with increasing (decreasing) values of $2 \theta$ (of $d$ ): at large $2 \theta$ values $\mathrm{R}_{i \mathrm{t}}$ and $R_{i \mathrm{~d}}$ are weakly correlated for the two wavelengths. $\mathrm{RCS}_{i}$ lies around zero for both wavelengths, which indicates that $R_{i \mathrm{t}}$ and $R_{i \mathrm{~d}}$ are on a similar scale, except for some $2 \theta$ intervals where

\section{Postmortem analysis}

It may be worth analyzing a posteriori the reasons conditioning the failure or the success of the method in order to derive useful suggestions for future work. We first notice that while accurate intensity measurements for single-crystal data are mandatory for the success of single-wavelength anomalous dispersion-multiple-wavelength anomalous dispersion (SADMAD) techniques (relative errors close to $5 \%$ may be critical), the need for such accuracy seems less severe for powder data if one takes into account the fact that the full pattern decomposition introduces large errors in the moduli estimates. Indeed the crystallographic residual between the true moduli and the moduli obtained by full pattern decomposition is usually large, depending on the structure size and complexity, the space-group symmetry, the experimental diffraction technique and the measurement angle $2 \theta$. To clarify the question we calculate for each pair of variables $(x, y)$ two types of residual,

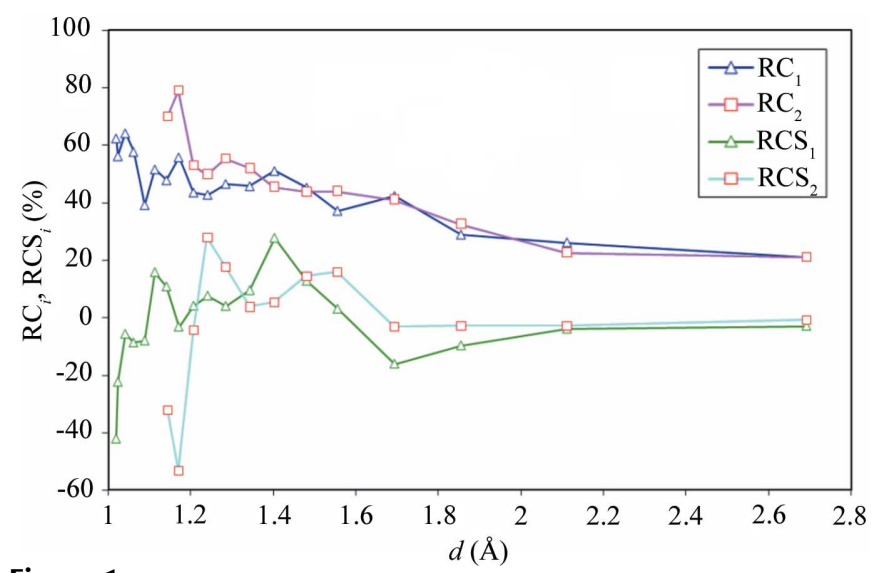

Figure 1

$\mathrm{RC}_{1}, \mathrm{RC}_{2}, \mathrm{RCS}_{1}$ and $\mathrm{RCS}_{2}$ versus the interplanar spacing $d$ for PLAT4 for each batch of 100 reflections sharing the same $2 \theta$ interval. systematic overestimation or underestimation of one of the two variables is made.

In Fig. 2 we show the residuals $\mathrm{RC}_{\Delta}$ and $\mathrm{RCS}_{\Delta}$ for the variables $y=\left(R_{2 \mathrm{t}}-R_{1 \mathrm{t}}^{\prime}\right)$ and $x=\left(R_{2 \mathrm{~d}}-R_{1 \mathrm{~d}}^{\prime}\right)$ [see equation (9)]. $\mathrm{RC}_{\Delta}$ is always far from zero and increases with increasing values of $2 \theta$. Since the estimate of $\varphi_{1}$ depends on the value of $\left(R_{2 \mathrm{~d}}-R_{1 \mathrm{~d}}^{\prime}\right)$ [i.e. if the sign of $\left(R_{2 \mathrm{~d}}-R_{1 \mathrm{~d}}^{\prime}\right)$ changes, then the $\varphi_{1}$ estimate changes by $\pi$ ], the dramatically large values of $\mathrm{RC}_{\Delta}$ should not allow good phase estimates, in contrast with the success of our procedure. To explain this contradictory feature, we show in Fig. 2 the percentage (\%inv) of reflections (per batch) for which the sign of $\left(R_{2 \mathrm{~d}}-R_{1 \mathrm{~d}}^{\prime}\right)$ is opposite to that of $\left(R_{2 \mathrm{t}}-R_{1 \mathrm{t}}^{\prime}\right)$. We see that, in spite of the large $R C_{\Delta}$ values, a remarkable sign inversion occurs only for two batches: those corresponding to the $d \simeq 1 \AA$ and $d \simeq 1.4 \AA$ intervals where $\mathrm{RCS}_{2}$ is far from zero. This coincidental occurrence suggests that both the lack of experimental information at highresolution $2 \theta$ intervals and a wrong background estimate may be responsible for the phase error.

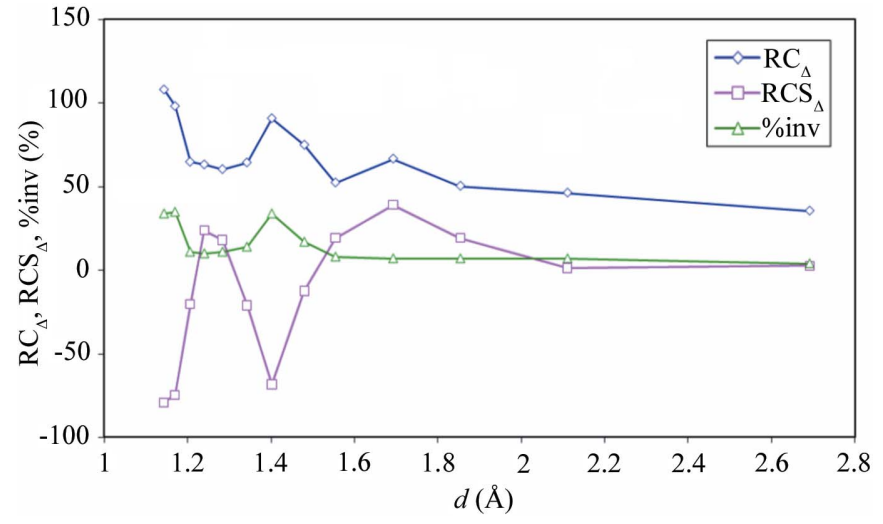

Figure 2

$\mathrm{RC}_{\Delta}, \mathrm{RCS}_{\Delta}$ and \%inv for PLAT4 for each batch of 100 reflections sharing the same $2 \theta$ interval. 
Table 3

Cumulative distributions of the phase error versus ARG for PLAT4.

In columns 2 to 5 equations (2) and (3), simplified via equation (9), are applied. The columns 'Negative relationships' and 'Positive relationships' refer to reflections for which $G$ [and therefore $\left\langle\cos \left(\varphi_{1}-\varphi_{3}\right)\right\rangle$ ] is negative or positive, respectively. For each value of ARG the number of relationships with $|G|>$ ARG (No.) and the corresponding average phase error $\langle|\Delta \varphi|\rangle$ are given. In columns 6 to 9 equations (5) to (7), simplified via equations (10), are applied. The columns 'Negative relationships' and 'Positive relationships' refer to reflections for which $S$ [and therefore $\left.\left\langle\cos \left(\varphi_{1}-\varphi_{3}\right)\right\rangle\right]$ is negative or positive, respectively. For each value of ARG the number of relationships with $|S|>$ ARG (No.) and the corresponding average phase error $\langle|\Delta \varphi|\rangle$ are given.

\begin{tabular}{|c|c|c|c|c|c|c|c|c|}
\hline \multirow[b]{3}{*}{ ARG } & \multicolumn{4}{|c|}{ Equations (2) and (3) } & \multicolumn{4}{|c|}{ Equations (5) to (7) } \\
\hline & \multicolumn{2}{|c|}{ Negative relationships } & \multicolumn{2}{|c|}{ Positive relationships } & \multicolumn{2}{|c|}{ Negative relationships } & \multicolumn{2}{|c|}{ Positive relationships } \\
\hline & No. & $\langle|\Delta \varphi|\rangle\left(^{\circ}\right)$ & No. & $\langle|\Delta \varphi|\rangle\left(^{\circ}\right)$ & No. & $\langle|\Delta \varphi|\rangle\left(^{\circ}\right)$ & No. & $\langle|\Delta \varphi|\rangle\left(^{\circ}\right)$ \\
\hline 2.0 & 815 & 1 & 44 & 180 & 938 & 2 & 8 & 180 \\
\hline 4.0 & 592 & 0 & 24 & 180 & 785 & 0 & 3 & 180 \\
\hline 6.0 & 397 & 0 & 18 & 180 & 627 & 0 & & \\
\hline 8.0 & 265 & 0 & 13 & 180 & 495 & 0 & & \\
\hline 14.0 & 34 & 0 & & & 143 & 0 & & \\
\hline 16.0 & 18 & 0 & & & 78 & 0 & & \\
\hline
\end{tabular}

In Fig. 3(a) we show, for PLAT4, the $2 \theta$ interval $37.5-39.9^{\circ}$, corresponding to $1.48-1.40 \AA$ in terms of resolution, of the $\lambda_{1}$ PLAT4 experimental powder diffraction profile. The blue line describes the polynomial representation of the background as automatically estimated by the modified version of EXPO2004. The vertical blue bars in the lower part of the figure correspond to the positions of the reflections falling in that interval. It is evident that the background model is not accurate: its overestimation causes the underestimation of the reflection intensities obtained by profile decomposition, with consequent large errors in the $R_{1 \mathrm{~d}}$ estimates.

In Fig. 3(b) we show the $2 \theta$ interval $52.0-55.9^{\circ}$, corresponding to $1.22-1.44 \AA$ resolution, of the $\lambda_{2}$ PLAT4 experimental powder diffraction profile. Owing to peak overlapping, uncertainty of the background and atomic scattering decay, the signal is very noisy: large errors on the $R_{2 \mathrm{t}}$ estimates are expected, as statistically testified in Fig. 1.

From the above observations the following clues may be obtained:

(a) Synchrotron radiation plays a fundamental role in this technique, because it allows wavelength tuning and reduces the peak overlapping.

(b) The systematic overestimation or underestimation of the diffraction intensities does not necessarily lead to a sign inversion [i.e. when the sign of $\left(R_{2 \mathrm{~d}}-R_{1 \mathrm{~d}}^{\prime}\right)$ is opposite to that of $\left(R_{2 \mathrm{t}}-R_{1 \mathrm{t}}^{\prime}\right)$ ]. Quite frequently the full pattern decomposition mechanism overestimates or underestimates reflection intensities at both wavelengths, owing to the similarities of the two experimental diffraction profiles. As a consequence, moderately large values of $\mathrm{RC}_{\Delta}$ are still compatible with good phase estimates.

(c) The correctness of the background modelling is much more critical for multi-wavelength than for one-wavelength powder crystallography. Indeed, in the first case the correctness of the phase assignment may depend on quite small intensity differences rather than on the full intensities.

(d) The signal-to-noise ratio should be carefully evaluated before deciding which experimental $2 \theta$ interval should be included in the calculations. We checked that of the 34 positive relationships with $G>1.4$ wrongly estimated via equations (2) and (3), six fall in the interval shown in Fig. 3(a) and 27 belong to the final part of the experimental profile, shown in Fig. $3(b)$.

(e) Since large peak overlapping and smaller signal-to-noise ratio weaken the efficiency of the full pattern decomposition,

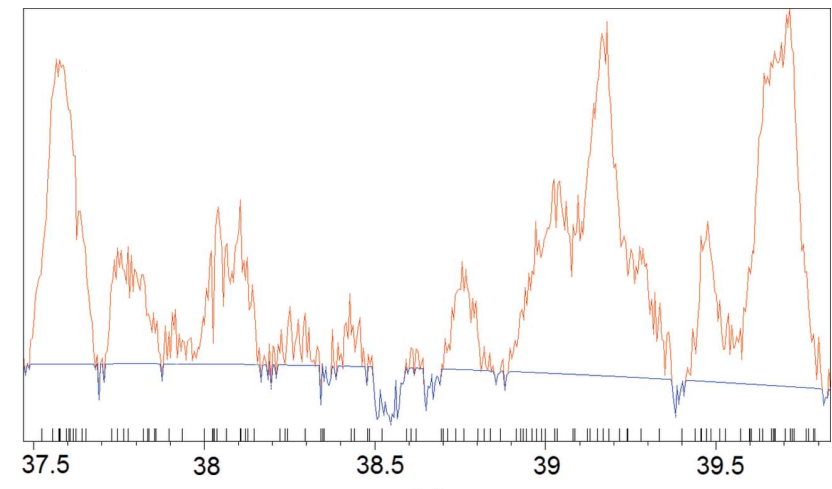

(a)

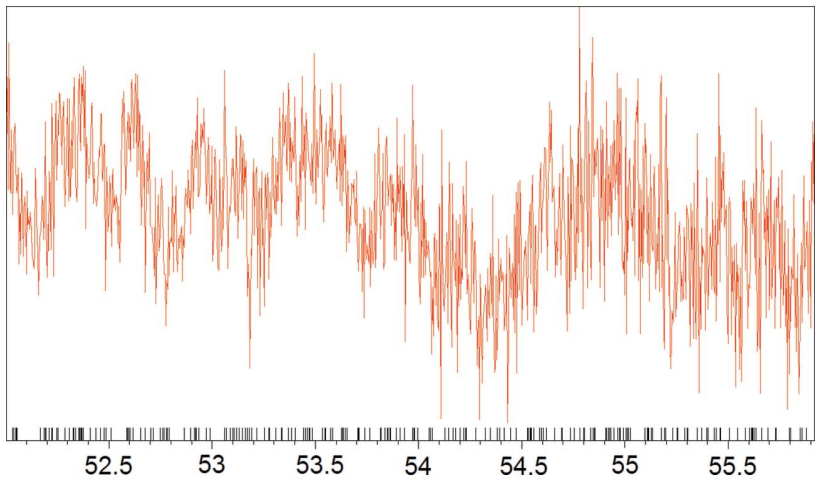

(b)

Figure 3

Experimental powder diffraction profiles for PLAT4 for: $(a)$ the $2 \theta$ interval $37.5-39.9^{\circ}$, corresponding to $1.40-1.48 \AA$ in terms of resolution ( $\lambda_{1}$ wavelength); $(b)$ the final $2 \theta$ interval $52.0-55.9^{\circ}$, corresponding to $1.22-1.44 \AA$ resolution ( $\lambda_{2}$ wavelength). The blue line shows the polynomial representation of the background. 
Table 4

Cumulative distributions of the phase error versus ARG for PLAT4 assuming $e_{i}=1.05\left[1+\left(\sin ^{2} \theta\right) / \lambda^{2}\right]$ for $i=1,2,3$.

See Tables 2 and 3 for details.

\begin{tabular}{|c|c|c|c|c|c|c|c|c|}
\hline \multirow[b]{3}{*}{ ARG } & \multicolumn{4}{|c|}{ Equations (2) and (3) } & \multicolumn{4}{|c|}{ Equations (5) to (7) } \\
\hline & \multicolumn{2}{|c|}{ Negative relationships } & \multicolumn{2}{|c|}{ Positive relationships } & \multicolumn{2}{|c|}{ Negative relationships } & \multicolumn{2}{|c|}{ Positive relationships } \\
\hline & No. & $\langle|\Delta \varphi|\rangle\left(^{\circ}\right)$ & No. & $\langle|\Delta \varphi|\rangle\left(^{\circ}\right)$ & No. & $\langle|\Delta \varphi|\rangle\left(^{\circ}\right)$ & No. & $\langle|\Delta \varphi|\rangle\left(^{\circ}\right)$ \\
\hline 1.0 & 613 & 0 & 18 & 180 & 791 & 0 & 1 & 180 \\
\hline 2.0 & 297 & 0 & 8 & 180 & 468 & 0 & & \\
\hline 3.0 & 129 & 0 & & & 251 & 0 & & \\
\hline 4.0 & 47 & 0 & & & 118 & 0 & & \\
\hline
\end{tabular}

an angle-dependent weighting scheme may be useful. We modified the error parameters $e_{i}, i=1,2,3$, assumed to be constant in the default applications described in $\S 6$, according to the weighting scheme

$$
e_{i}=1.05\left[1+\left(\sin ^{2} \theta\right) / \lambda^{2}\right] \text { for } i=1,2,3 .
$$

We extended $i$ up to 3 because the substructure model is expected to provide larger errors for high-resolution reflections. The integration of equation (11) into equation (9) leads to Table 4, where the cumulative distribution of the phase error versus $|G|$ is shown. Comparing it with the distribution shown in Table 3 suggests that the wrong phase estimates are now confined to smaller ARG values. For example, according to Table 3 there are 13 wrong phase estimates among the most reliable 265 phase relationships: in Table 4 there are only 8 wrong phase estimates among the most reliable 297 phase relationships.

Two other additional features condition the success of the procedure:

(i) The (resolution dependent) ratio $\sum_{3} / \sum_{1}$, decisive for finding the substructure: it was very favourable for both our test cases, for which $|\Delta f| \geq 8$. In cases where the anomalous scatterers are not as heavy and the structure size is larger, the ratio $\sum_{3} / \sum_{1}$ may become critical.

(ii) The ratio 'scattering power of the anomalous substructure/scattering power of the full structure': in both our test cases this ratio was particularly high, but it becomes critical when it is relatively small. Indeed, in this last case the experimental noise may overcome the signal, with a consequent increase in the percentage of sign inversions.

We leave this study to future investigations.

\section{Conclusions}

The method of joint probability distribution functions has been successfully applied to two-wavelength powder data affected by anomalous dispersion: assumptions were made which allow one to disregard the $f^{\prime \prime}$ component. The mathematical approach provided two types of formulas for estimating the phases of a crystal structure when the anomalous substructure is known. The first type [equations (2) and (3)] requires an approximation that is not always guaranteed, the second [equations (5)-(7)] is expected to be valid in more general conditions. Both were simplified without loss of efficiency by introducing useful approximations in the errors. When applied to the experimental data of two crystal structures all the formulas provided useful phase estimations.

It is worth noting that since a general assumption allows one to neglect the $f^{\prime \prime}$ component, the final formulas derived above may be applied to isomorphous replacement.

\section{APPENDIX $A$ \\ Explicit expression for $e_{1} e_{2} e_{3} \operatorname{det}(\mathbf{L})$}

In order to derive simpler formulas for phase estimation we calculate the explicit expression of $e_{1} e_{2} e_{3} \operatorname{det}(\mathbf{L})$. We obtain

$$
\begin{aligned}
e_{1} e_{2} e_{3} \operatorname{det}(\mathbf{L}) & \\
= & e_{1} e_{2} e_{3}\left(e_{1} e_{2} e_{3} \sum_{1} \sum_{2} \sum_{3}-e_{1} \sum_{23}^{2} \sum_{1}-e_{2} \sum_{13}^{2} \sum_{2}\right. \\
& \left.-e_{3} \sum_{12}^{2} \sum_{3}+2 \sum_{12} \sum_{13} \sum_{23}\right) /\left(e_{1} e_{2} e_{3} \sum_{1} \sum_{2} \sum_{3}\right) \\
= & \left\{\sum _ { 1 } \sum _ { 3 } \left[e_{3}\left(e_{1} e_{2}-1\right) \sum_{1}+e_{1}\left(e_{2} e_{3}-1\right) \sum_{3}\right.\right. \\
& \left.+2\left(e_{1} e_{2} e_{3}-e_{1}-e_{3}+1\right) \sum_{13}\right]-\sum_{13}^{2}\left[\left(e_{1}+e_{2}-2\right) \sum_{1}\right. \\
& \left.\left.+\left(e_{2}+e_{3}-2\right) \sum_{3}+2\left(e_{2}-1\right) \sum_{13}\right]\right\} /\left(\sum_{1} \sum_{2} \sum_{3}\right) .
\end{aligned}
$$

Equation (12) clearly shows that $\operatorname{det}(\mathbf{L})$ vanishes when $e_{i}=0$ for $i=1,2,3$, as theoretically anticipated in the main text. Each of the terms in the numerator of equation (12) contains a polynomial which depends on the $e_{i}$ values: each polynomial is different from the others but all are non-negative definite, and all increase when the $e_{i}$ 's increase. Since $e_{i}$ 's are supposed to be small, we may approximate all the polynomials by a unique non-negative expression, e.g. $e_{3}\left(e_{1} e_{2}-1\right)$. Then equation (12) may be approximated by

$$
\begin{aligned}
e_{1} e_{2} e_{3} \operatorname{det}(\mathbf{L}) \simeq & \left(\sum_{1} \sum_{3}-\sum_{13}^{2}\right) e_{3}\left(e_{1} e_{2}-1\right) \\
& \times\left(\sum_{1}+\sum_{3}+2 \sum_{13}\right) /\left(\sum_{1} \sum_{2} \sum_{3}\right) \\
= & \left(\sum_{1} \sum_{3}-\sum_{13}^{2}\right) e_{3}\left(e_{1} e_{2}-1\right) \\
& \times \sum_{2} /\left(\sum_{1} \sum_{2} \sum_{3}\right) \\
= & e_{3}\left(e_{1} e_{2}-1\right) Z_{\mathrm{c}} \sum_{2} /\left(\sum_{1} \sum_{2} \sum_{3}\right),
\end{aligned}
$$

where

$$
Z_{\mathrm{c}}=\left(\sum_{1} \sum_{3}-\sum_{13}^{2}\right)
$$


We acknowledge the Paul Scherrer Institute for provision of the Swiss Light Source synchrotron radiation facility.

\section{References}

Altomare, A., Belviso, B. D., Burla, M. C., Campi, G., Cuocci, C., Giacovazzo, C., Gozzo, F., Moliterni, A., Polidori, G. \& Rizzi, R. (2009). J. Appl. Cryst. 42, 30-35.

Altomare, A., Caliandro, R., Camalli, M., Cuocci, C., Giacovazzo, C., Moliterni, A. G. G. \& Rizzi, R. (2004). J. Appl. Cryst. 37, 1025-1028.

Altomare, A., Cuocci, C., Giacovazzo, C., Moliterni, A. \& Rizzi, R. (2008). J. Appl. Cryst. 41, 592-599.

Altomare, A., Cuocci, C., Giacovazzo, C., Moliterni, A. G. G. \& Rizzi, R. (2006). J. Appl. Cryst. 39, 558-562.

Burla, M. C., Carrozzini, B., Cascarano, G. L., Giacovazzo, C. \& Polidori, G. (2003). Acta Cryst. D59, 662-669.
Burla, M. C., Carrozzini, B., Cascarano, G. L., Giacovazzo, C., Polidori, G. \& Siliqi, D. (2002). Acta Cryst. D58, 928-935.

David, W. I. F. (2004). J. Appl. Cryst. 37, 621-628.

Giacovazzo, C. (1979). Acta Cryst. A35, 757-764.

Giacovazzo, C. \& Siliqi, D. (2001). Acta Cryst. A57, 700-707.

Gu, Y. X., Liu, Y. D., Hao, Q. \& Fan, H. F. (2000). Acta Cryst. A56, 592-595.

Helliwell, J. R., Helliwell, M. \& Jones, R. H. (2005). Acta Cryst. A61, 568-574.

Janczak, J. \& Kubiak, R. (2002). Inorg. Chim. Acta, 342, 64-76.

Le Bail, A., Duroy, H. \& Fourquet, J. L. (1988). Mater. Res. Bull. 23, 447-452.

Main, P. (1978). Acta Cryst. A34, 31-38.

Prandl, W. (1990). Acta Cryst. A46, 988-992.

Prandl, W. (1994). Acta Cryst. A50, 52-55.

Rietveld, H. M. (1969). J. Appl. Cryst. 2, 65-71. 